\title{
Blood pressure variability in relation to autonomic nervous system dysregulation: the X-CELLENT study
}

\author{
Yi Zhang ${ }^{1,2}$, Davide Agnoletti ${ }^{1}$, Jacques Blacher ${ }^{1}$ and Michel E Safar ${ }^{1}$
}

The purpose of this study was to investigate the association of autonomic nervous system dysregulation with blood pressure variability. Among the 2370 participants in the X-CELLENT study, 577 patients ( $59.0 \pm 10.2$ years) were randomly selected to participate in an ancillary ambulatory blood-pressure monitoring study. We proposed a novel autonomic nervous system regulation index termed dSBP/dHR, which was defined as the steepness of the slope of the relationship between the 24-h systolic blood pressure (SBP) and the heart rate (HR) for each participant. Within-subjects s.d. of SBP, weighted for the time interval between consecutive validated readings from 24-h ambulatory blood pressure monitoring, was used to evaluate blood pressure variability. When dSBP/dHR was divided into tertiles, we observed a progressive increase from tertile 1 to tertile 3 in the daytime SBP, a progressive decrease in nighttime SBP, and consequently a progressive increase in the day-night SBP gradient $(P<0.001)$. The s.d. of both daytime and nighttime SBPs were consistently and significantly increased from tertile 1 to tertile $3(P<0.01)$. Both before and after adjustment for age, gender and 24-h mean blood pressure, all of these increasing and decreasing trends reached statistical significance $(P<0.01)$. Furthermore, in our sensitivity analysis, when men and women were considered separately, the findings remained unaltered. In summary, autonomic nervous system dysfunction was associated with a heightened day-night SBP gradient and more variable SBP over $24 \mathrm{~h}$ in patients with essential hypertension.

Hypertension Research (2012) 35, 399-403; doi:10.1038/hr.2011.203; published online 1 December 2011

Keywords: autonomic nervous system; blood pressure variability; systolic blood pressure

\section{INTRODUCTION}

There are several lines of evidence that indicate blood pressure variability (BPV) is a major determinant of cardiovascular (CV) events and mortality in various populations. ${ }^{1-4}$ Furthermore, some investigators have demonstrated that the nighttime BPV, assessed by s.d. of nighttime systolic blood pressure (SBP) using a 24-h ambulatory blood pressure monitor (ABPM), is more strongly associated with target organ damage than is daytime $\mathrm{BPV}^{5-7}$ From a physiological point of view, without the various daytime influences that affect the blood pressure (BP), such as food digestion and physical exercise, the BP is mainly regulated by the autonomic nervous system (ANS) during sleep. Moreover, it has recently been reported that impaired ANS regulation was significantly associated with an increased carotid intima-media thickness. ${ }^{8}$ Because both nighttime BPV and ANS dysregulation are significantly associated with target organ damage, it would be interesting to know whether ANS dysregulation could be the cause of the increased BP variation and consequently has an impact on target organ damage. However, data are limited with regard to the association of ANS regulation with BPV. Accordingly, we proposed a novel ANS regulation index that is calculated by conventional 24-h ABPM, and investigated its potential relationship with daytime and nighttime BPV in the X-CELLENT study.

\section{METHODS}

Study design

The X-CELLENT study (NatriliX SR vs. CandEsartan and amLodipine in the reduction of systoLic blood prEssure in hyperte-Nsive patienTs) is a multicenter, multinational, randomized, double-blinded, and placebo-controlled study with four parallel treatment arms (placebo, indapamide, candesartan and amlodipine). In total, 2370 outpatients (aged $40-80$ years) with essential hypertension were recruited. The inclusion criteria included $150 \mathrm{~mm} \mathrm{Hg} \leqslant \mathrm{SBP}<$ $180 \mathrm{~mm} \mathrm{Hg}$ and $95 \mathrm{~mm} \mathrm{Hg} \leqslant$ diastolic blood pressure (DBP) $<110 \mathrm{~mm} \mathrm{Hg}$ or $160 \mathrm{~mm} \mathrm{Hg} \leqslant \mathrm{SBP}<180 \mathrm{~mm} \mathrm{Hg}$ and $\mathrm{DBP}<90 \mathrm{~mm} \mathrm{Hg}$. The exclusion criteria included a history of coronary artery disease, heart failure, stroke or transient ischemic attack, left ventricular hypertrophy, diabetes mellitus (type 1 or type 2), and renal failure. More details concerning the X-CELLENT study can be found in our previous publications. ${ }^{9}$ From the X-CELLENT study population, 577 patients (aged $59.0 \pm 10.2$ years) were randomly selected to participate in an ancillary ABPM study. Furthermore, because the effects of treatment were withheld from the present analysis, only the baseline data were present. The local Ethics Committee approved the study protocol and written informed consent was obtained from each study participant.

Anthropometric and BP measurements

Body weight and body height were measured for each subject at the inclusion, and the body mass index was calculated as the body weight in $\mathrm{kg}$ divided by the

${ }^{1}$ Paris Descartes University; AP-HP; Diagnosis and Therapeutic Center, Hôtel-Dieu, Paris, France and ${ }^{2}$ Centre for Epidemiological Studies and Clinical Trials, Ruijin Hospital, Shanghai Jiaotong University School of Medicine, Shanghai, China

Correspondence: Dr ME Safar, Centre de Diagnostic et de Thérapeutique, Hôtel-Dieu, 1, place du Parvis Notre-Dame, 75181 Paris Cedex 04, France. E-mail: michel.safar@htd.aphp.fr

Received 24 January 2011; revised 20 September 2011; accepted 22 September 2011; published online 1 December 2011 
square of the body height in meters. After at least $5 \mathrm{~min}$ of rest in the sitting position, BP was measured three times with a validated oscillometric BP monitor (Omron 705CP, Kyoto, Japan), and those readings were averaged for further analysis.

\section{ABPM}

The ABPM was performed on 577 participants according to the recommendations of the European Society of Hypertension. ${ }^{10}$ The frequency of ABPM capture was every $15 \mathrm{~min}$ over the entire $24 \mathrm{~h}$. Patients' wakeful and sleeping time were self-reported and recorded for further calculation. The daytime and nighttime were defined as the time from waking until sleep and from sleep until waking, respectively, for each participant.

To minimize the effects of recording errors during the 24-h ABPM, we used the within-subject mean and s.d., weighted for the time interval between consecutive validated readings, to evaluate the mean BP level and variability. Considering the nighttime dipping effect, the mean and s.d. were calculated for the awake and sleeping periods, as daytime mean and s.d. and nighttime mean and s.d., respectively.

We also examined the relationship between SBP ( $y$-axis) and heart rate (HR) ( $x$-axis) on the basis of the 24-h SBP-HR correlation plots for each participant. Then, the steepness of the slope of the relationship between SBP and HR was calculated as $\mathrm{dSBP} / \mathrm{dHR}$, an ambulatory index representing the change in SBP per 1-unit change in $\mathrm{HR}\left(\mathrm{mm} \mathrm{Hg}\right.$ per beat $\left.\mathrm{min}^{-1}\right)$. For example, as shown in Figure 1, with a 10 beat $\mathrm{min}^{-1}$ increase in $\mathrm{HR}$, patient $\mathrm{B}$ is expected to have an increase in SBP of $7.5 \mathrm{~mm} \mathrm{Hg}$, whereas patient A would have an increase of $13.1 \mathrm{~mm} \mathrm{Hg}$.

The day-night SBP gradient was defined as the difference in SBP between day and night.

\section{Statistical analysis}

Quantitative and qualitative parameters are presented as the mean \pm s.d. and numbers with percentages in parentheses, respectively. Individual correlation plots were performed by a simple linear regression between 24-h SBP and HR. Next, the dSBP/dHR term was defined as the steepness of the slope of the relationship between SBP and HR in each participant. We applied the ANOVA and chi-square tests to compare quantitative and qualitative variables, respectively, between subjects in different groups classified by tertiles of dSBP/dHR. Similar analyses were performed to compare the mean SBP, SBP s.d. and daynight SBP gradient, and their trends were tested in a linear model after adjustment for age, gender and 24-h mean BP, which were all significantly associated with the $\mathrm{dSBP} / \mathrm{dHR}$ in the univariate analysis. Sensitivity analyses were conducted in men and women separately. The statistical analysis was performed using SAS software, version 9.1 (SAS Institute, Cary, NC, USA). $P<0.05$ was considered statistically significant.

\section{RESULTS}

\section{Characteristics of participants}

Table 1 shows the characteristics of the participants classified into tertiles by dSBP/dHR. There was no significant association of the dSBP/dHR with the participants' baseline characteristics, with the exception of male gender and 24-h BP. Specifically, the proportion of men increased significantly $(P=0.02)$, with $46.6,44.0$ and $57.4 \%$ in tertiles 1, 2 and 3 of dSBP/dHR, respectively, and the increasing trend of 24-h mean SBP was marginally significant from tertile 1 to tertile 3 of $\mathrm{dSBP} / \mathrm{dHR} \quad(P=0.05)$, with $141.4 \pm 13.3,139.6 \pm 11.8$ and $142.7 \pm 13.1 \mathrm{~mm} \mathrm{Hg}$ in tertiles 1,2 and 3 of $\mathrm{dSBP} / \mathrm{dHR}$, respectively.

\section{Association of autonomic dysregulation with the nocturnal BP dipping pattern}

The nocturnal BP dipping pattern and its association with $\mathrm{dSBP} / \mathrm{dHR}$ were also investigated. The proportions of Risers (the nighttime SBP is greater than daytime SBP), Non-dippers (the nighttime SBP decreases between 0 and $10 \%$ compared with daytime SBP), Dippers (the nighttime SBP decreases between 10 and 20\%) and Extreme-dippers (the nighttime SBP decreases more than 20\%) were 10.4, 34.0, 47.0 and $8.6 \%$, respectively. The incidence of Riser and Non-dipper decreased, whereas the incidence of Dipper and Extreme-dipper increased progressively and significantly from tertile 1 to tertile 3 $(P<0.001$, Table 2$)$.

Association of autonomic dysregulation with BP and BP variability As shown in Table 2, when the dSBP/dHR was used to divide the subjects into tertiles, we observed a progressive increase in the daytime SBP, a progressive decrease in the nighttime SBP, and consequently, a progressive increase in the day-night SBP gradient, from tertile 1 to tertile $3(P<0.001)$. Moreover, the daytime and nighttime SBP s.d. were consistently and significantly increased from tertile 1 to tertile 3 $(P<0.01)$. Both before and after adjustment for age, gender and 24-h mean SBP, all of these increasing or decreasing trends reached statistical significance $(P<0.01)$. These findings were confirmed in our sensitivity analyses of men and women, separately (Figures 2 and 3 ).

Furthermore, the steepness of the slope between 24-h DBP and HR, termed $\mathrm{dDBP} / \mathrm{dHR}$, was calculated with the same algorithm as the $\mathrm{dSBP} / \mathrm{dHR}$. When the $\mathrm{dDBP} / \mathrm{dHR}$ was divided into tertiles, with values of $0.015-0.428 \mathrm{~mm} \mathrm{Hg}$ per beat $\mathrm{min}^{-1}$ for tertile $1,0.429$ $0.650 \mathrm{~mm} \mathrm{Hg}$ per beat $\mathrm{min}^{-1}$ for tertile 2 and $0.651-1.914 \mathrm{~mm} \mathrm{Hg}$ per beat $\mathrm{min}^{-1}$ for tertile 3 , the results were similar to those observed with
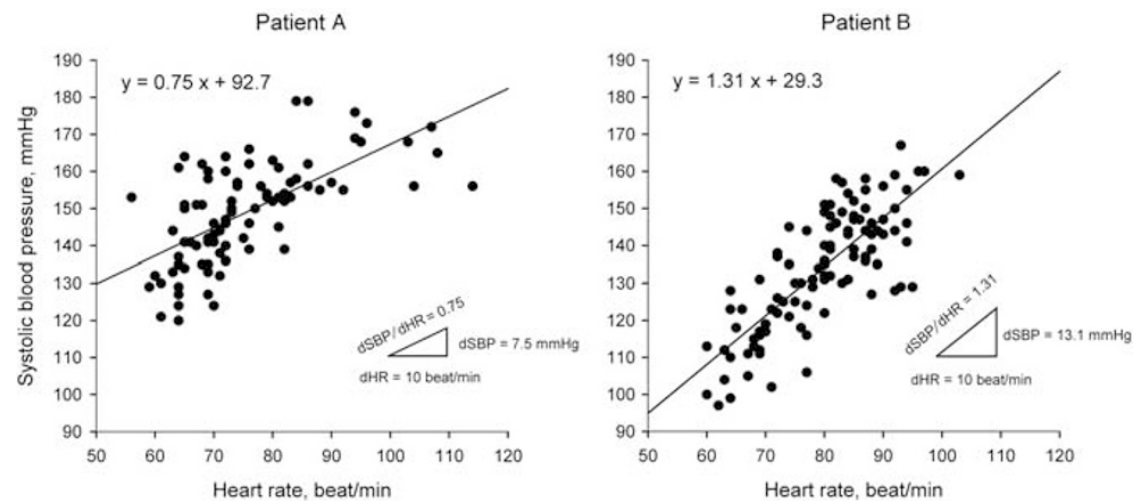

Figure 1 The definition of dSBP/dHR. Correlation plots between SBP and HR from the 24-h ambulatory blood pressure monitor are presented for patients $A$ and B. The dSBP/dHR indicates the steepness of the slope of the relationship between the 24-h SBP ( $y$-axis) and the HR ( $x$-axis) for each subject. For example, for a 10 beat $\mathrm{min}^{-1}$ change in $\mathrm{HR}$, the SBP increased by $7.5 \mathrm{~mm} \mathrm{Hg}$ in patient $\mathrm{A}$ and by $13.1 \mathrm{~mm} \mathrm{Hg}$ in patient $\mathrm{B}$. 
Table 1 Characteristics of participants in subjects with different tertiles of dSBP/dHR

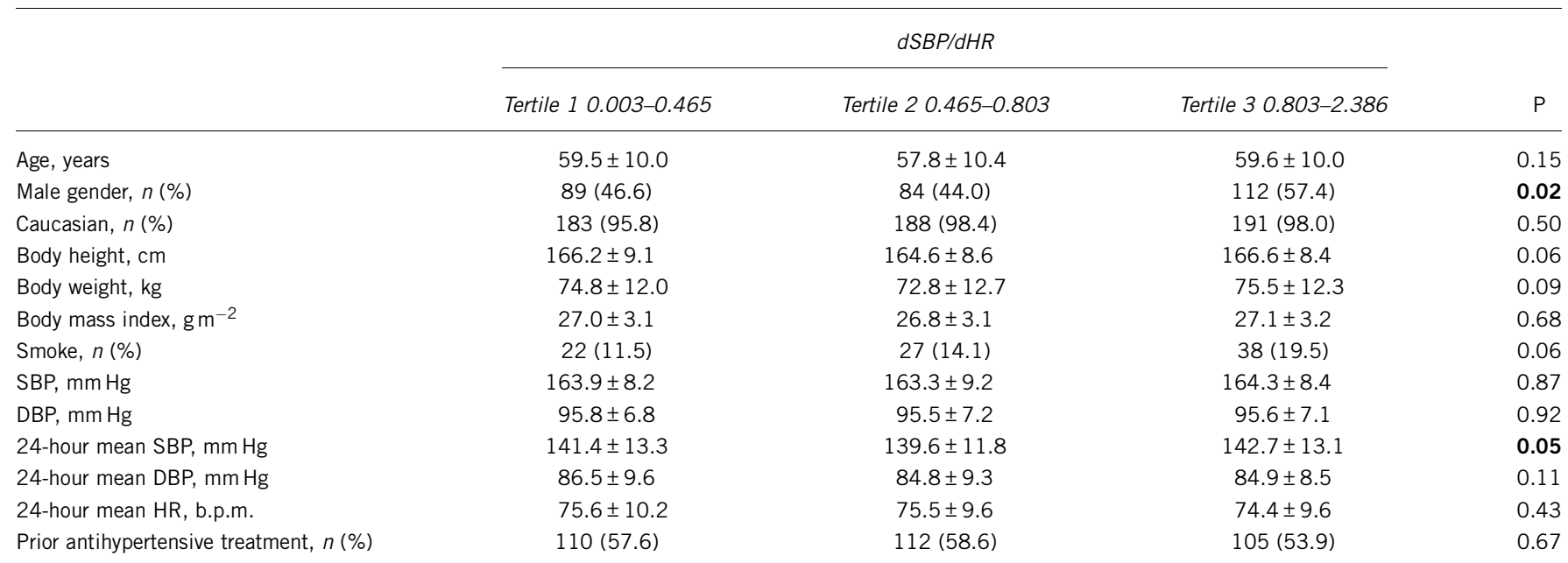

Abbreviations: b.p.m., beats per minute; DBP, diastolic blood pressure; HR, heart rate; SBP, systolic blood pressure.

Values are presented as mean \pm s.d. or number with percentage in parenthesis. Smoke indicates the current smoking; dSBP/dHR indicates the steepness of the slope of the relationship between 24-hour SBP (y-axis) and HR (x-axis) for each subject.

Table 2 Comparisons of daytime and nighttime SBP and blood pressure variability between tertiles of dSBP/dHR

\begin{tabular}{|c|c|c|c|c|c|}
\hline & \multicolumn{3}{|c|}{$d S B P / d H R$} & \multirow[b]{2}{*}{$\mathrm{P}$} & \multirow[b]{2}{*}{$P^{*}$} \\
\hline & Tertile $10.003-0.465$ & Tertile $20.465-0.803$ & Tertile $30.803-2.386$ & & \\
\hline Nighttime SBP, mm Hg & $134.7 \pm 16.2$ & $127.7 \pm 13.0$ & $127.8 \pm 15.6$ & $<0.001$ & $<0.001$ \\
\hline Day-night SBP gradient, $\mathrm{mm} \mathrm{Hg}$ & $9.7 \pm 11.4$ & $16.8 \pm 7.7$ & $22.2 \pm 10.5$ & $<0.001$ & $<0.001$ \\
\hline Riser pattern, $n(\%)$ & $32(53.3)$ & $20(33.3)$ & $8(13.3)$ & $<0.001$ & $<0.001$ \\
\hline Extreme-dipper pattern, $n(\%)$ & $5(10.0)$ & $9(18.0)$ & $36(72.0)$ & $<0.001$ & $<0.001$ \\
\hline Daytime s.d., mm Hg & $12.6 \pm 3.3$ & $12.5 \pm 3.3$ & $13.6 \pm 3.4$ & 0.002 & 0.007 \\
\hline Nighttime s.d., $\mathrm{mm} \mathrm{Hg}$ & $10.5 \pm 3.2$ & $10.4 \pm 2.8$ & $11.8 \pm 3.5$ & $<0.001$ & $<0.001$ \\
\hline
\end{tabular}

Abbreviations: HR, heart rate; SBP, systolic blood pressure

Values are mean \pm s.d. $P^{*}$ indicated the $P$ for trend after adjustment for age, sex and mean blood pressure. Daytime s.d. indicates time-weighted s.d. of SBP during awake time; Nighttime s.d. indicates time-weighted s.d. of SBP during sleeping time; Daytime and nighttime SBP are calculated with same algorithm; Day-night SBP gradient indicates the difference between daytime and nighttime SBP. Riser, non-dipper, dipper and extreme-dipper pattern was defined as nighttime SBP decreases greater than daytime SBP, and nighttime SBP decreases between 0\% and 10\%, between $10 \%$ and $20 \%$, and over 20\%, respectively, as compared with daytime BP. dSBP/dHR indicates the steepness of the slope of the relationship between 24 -hour SBP (y-axis) and HR (x-axis) for each subject.
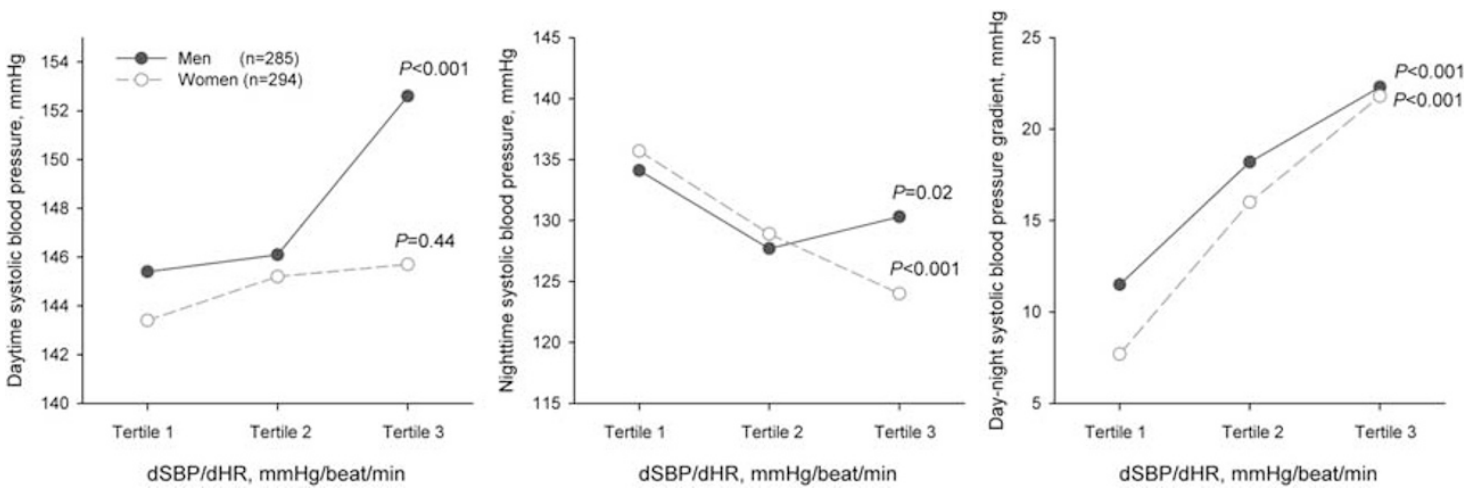

Figure 2 The association of the daytime and nighttime SBP and the day-night SBP gradient with tertiles of dSBP/dHR by gender. Men are represented by filled circles with solid lines, and women are represented by unfilled circles with dashed lines. The dSBP/dHR indicates the steepness of the slope of the relationship between the 24-h SBP ( $y$-axis) and the HR ( $x$-axis) for each subject and is studied in tertiles. The day-night SBP gradient indicates the difference between the daytime and nighttime SBP. A full color version of this figure is available at the Hypertension Research journal online. 

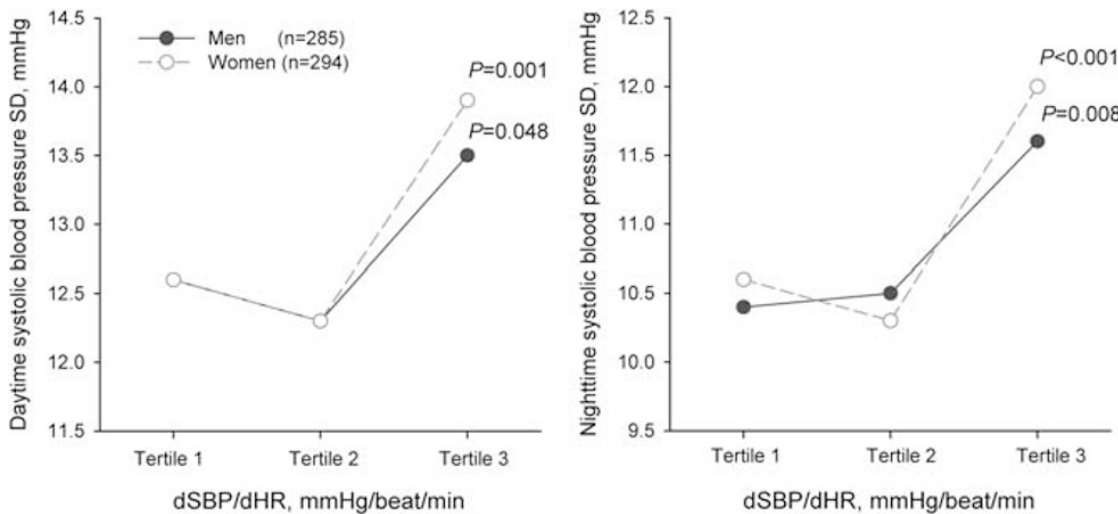

Figure 3 The association of the daytime and nighttime SBP s.d. with the tertiles of dSBP/dHR by gender. Men are represented by filled circles with solid lines, and women are represented by unfilled circles with dashed lines. The dSBP/dHR indicates the steepness of the slope of the relationship between the 24-h SBP (y-axis) and the HR (x-axis) for each subject and is studied in tertiles. The daytime s.d. indicates the time-weighted s.d. of the SBP during wakefulness; the nighttime s.d. indicates the time-weighted s.d. of the SBP during sleep. A full color version of this figure is available at the Hypertension Research journal online.

the SBP. The daytime DBP increased; however, the nighttime DBP decreased progressively and significantly from tertile 1 to 3 of the $\mathrm{dDBP} / \mathrm{dHR} \quad(P<0.001)$, with values of $88.9 \pm 9.9$, $89.9 \pm 9.4,90.6 \pm 8.8 \mathrm{~mm} \mathrm{Hg}$ for daytime DBP and $79.7 \pm 10.3$, $76.6 \pm 9.7,72.4 \pm 9.2 \mathrm{~mm} \mathrm{Hg}$ for nighttime DBP in tertiles 1,2 and 3 , respectively. Furthermore, both before and after adjustment for age, gender and 24-h mean DBP, the daytime and nighttime DBP s.d. increased consistently and significantly from tertile 1 to tertile $3(P<0.001)$, with values of $9.4 \pm 2.4,9.4 \pm 2.3$, $10.1 \pm 2.5 \mathrm{~mm} \mathrm{Hg}$ for the daytime DBP s.d. and $8.2 \pm 2.3,8.4 \pm 2.4$, $9.5 \pm 2.6 \mathrm{~mm} \mathrm{Hg}$ for the nighttime DBP s.d. in tertiles 1,2 and 3, respectively.

\section{DISCUSSION}

The main findings of the present study are twofold. First, ANS dysregulation, as evaluated with a novel index termed the dSBP/ dHR derived from 24-h ABPM, was significantly associated with an increase in the daytime SBP, a decrease in the nighttime SBP, and therefore an increase in the day-night SBP gradient. Second, ANS regulation was also significantly associated with increased daytime and nighttime BPVs when evaluated by the s.d. of daytime and nighttime SBP. The sensitivity analysis confirmed these two findings in men and women, separately.

Based on readings of 24-h ABPM, we proposed a novel ambulatory index that represents the amplitude of change in SBP per 1-unit change in HR. This means that for a given change in $\mathrm{HR}$, subjects with a higher $\mathrm{dSBP} / \mathrm{dHR}$ are expected to have a greater modification in SBP than subjects with a lower dSBP/dHR. Similarly, Coats et al. ${ }^{11}$ proposed another index calculated as the slope of the relationship between the R-R interval ( $y$-axis) and the SBP ( $x$-axis), and reported that it was inversely correlated with the baroreflex sensitivity as evaluated with the phenylephrine method $(r=-0.55, P<0.001)$. A more complicated methodology involving spectral analysis of the R-R interval and the SBP variability was employed by Lucini et al. to investigate the impaired ANS regulation in hypertensive patients. ${ }^{12,13}$ Compared with those parameters, our $\mathrm{dSBP} / \mathrm{dHR}$ is the simplest index and is conveniently derived from 24-h ABPM, thereby rendering it more pragmatic for clinical practice.
In the literature, the relationship between $\mathrm{HR}$ and $\mathrm{BP}$ has been studied on conscious rats before and after spinal cord transaction ${ }^{14}$ as a model in which the SBP-HR relationship indicates the autonomic nervous function. From a physiological point of view, the existing SBP-HR relationship could reflect not only the baroreflex sensitivity, but also sympathetic and parasympathetic cardiac control and vasomotor modulation. Therefore, the dSBP/dHR from the 24-h ABPM could be considered to be a robust yet practical index that represents the magnitude of CV ANS regulation. Moreover, we also found that the $\mathrm{dDBP} / \mathrm{dHR}$, another ANS regulation index derived with a similar algorithm, was also positively and significantly associated with BP and variability in the present study. This finding confirmed that ANS dysregulation, whether assessed by changes in the SBP or the DBP per unit change in HR, had a significant influence on BP and its variability. Furthermore, Eguchi et al. ${ }^{15}$ reported that some ANS regulation indices, such as the nighttime HR variability and the HR-SBP relationship from 24-h ABPM, could significantly predict CV events in diabetic patients.

In this study, when the $\mathrm{dSBP} / \mathrm{dHR}$ was divided into 3 tertiles, the subjects with the highest $\mathrm{dSBP} / \mathrm{dHR}$ (tertile 3) also had the highest daytime SBP, the lowest nighttime SBP, and the largest daynight SBP gradient. From a pathophysiological viewpoint, this finding may be attributable to overactive sympathetic control (in the daytime) and overactive vagal control (in the nighttime) of the cardiac and vascular regulation system. Furthermore, subjects with higher dSBP/dHR values are prone to have a more variable SBP in both the daytime and the nighttime, indicating that the ANS dysregulation also contributes to the increased daytime and nighttime BPV. Ichihara et al. ${ }^{16}$ reported a positive relationship between the BPV and arterial stiffness as assessed by pulse wave velocity in patients with hypertension, whereas in this study, we indicated that the BPV was also significantly associated with ANS dysregulation in both men and women. Further studies are warranted to elucidate whether arterial stiffening and ANS dysregulation synergistically or separately influence the arterial BPV. Moreover, in a meta-analysis, Webb et al. ${ }^{17}$ summarized that calcium channel blockers and non-loop diuretics could effectively reduce the BPV in hypertensive patients. However, whether the effectiveness of these two agents on the BPV 
control is due to arterial de-stiffening or to a re-balancing of the sympathetic and vagal CV regulation remains unknown. The underlying mechanisms need to be revealed by further fundamental studies.

It is counterintuitive that the dipper pattern (an increase in the day-night SBP gradient) is normally considered to a healthy regulation of BP, whereas increased beat-to-beat BPV always indicates an unhealthy BP regulation. This is a paradox, because the lowering of BP is often associated with an increase in BPV. For example, in this study, we found that the $\mathrm{dSBP} / \mathrm{dHR}$ index of ANS regulation was associated with an increased BP and variability $(P<0.001)$, but also with an increased incidence of the dipper pattern $(P<0.001)$. Theoretically, the dipper pattern is defined by a presumption that the nighttime SBP should decrease $10-20 \%$ due to dominant vagal CV control at night, which suggests that this index is more sensitive to nighttime ANS regulation. In contrast, the $\mathrm{dSBP} / \mathrm{dHR}$ is defined as the slope of regression plots of the 24-h BP recordings, which reflect 24-h BP regulation that includes not only the dominant nighttime vagal control but also the dominant daytime sympathetic control. This also helps to explain the observation that when both daytime and nighttime SBP are taken into account, the incidence of the extremedipper pattern is positively and significantly associated with the dSBP/ dHR $(P<0.001)$.

Our findings should be interpreted within the context of their limitations. First, the major limitation of this study is the lack of a validation test of the $\mathrm{dSBP} / \mathrm{dHR}$ in assessing ANS regulation to compare it with other classical indices, such as the HR variability, the ratio between the R-R interval and SBP (baroreflex sensitivity), or serum catecholamine levels. However, the aim of this study is not to validate another index reflecting ANS regulation in a hemodynamic laboratory, but to propose a novel index that can be conveniently derived from a 24-h ABPM recording and is associated with daytime and nighttime BP variabilities, as well as with the BP itself. Our sensitivity analysis, conducted in men and women separately, also confirmed these finding. Further study is still warranted to test the accuracy of this index in reflecting ANS regulation. Second, because this is a cross-sectional study, we are not able to distinguish the cause and consequence of any two related factors; however, as an ongoing prospective clinical trial, future data would provide valuable information in this respect.

In summary, we found that ANS dysregulation as evaluated by a simple ambulatory index derived from a $24-\mathrm{h}$ ABPM was significantly associated with an increased daytime SBP, a decreased nighttime SBP, and a consequently increased day-night SBP gradient, as well as with a more variable SBP in both the daytime and the nighttime in hypertensive patients.

\section{ACKNOWLEDGEMENTS}

We thank all of the investigators and the patients who participated in the $\mathrm{X}$-CELLENT study. We are grateful to the monitors and the staff of the Institut de Recherches Internationales Servier, France (Dr Luc Feldmann, Dr Martine de Champvallins, Dr Benoit Tallot, Mr Corentin Le Camus and Mrs Elsa Merkling)

1 Kikuya M, Hozawa A, Ohokubo T, Tsuji I, Michimata M, Matsubara M, Ota M, Nagai K, Araki T, Satoh H, Ito S, Hisamichi S, Imai Y. Prognostic significance of blood pressure and heart rate variabilities: the Ohasama study. Hypertension 2000; 36: 901-906.

2 Bjorklund K, Lind L, Zethelius B, Berglund L, Lithell H. Prognostic significance of 24-h ambulatory blood pressure characteristics for cardiovascular morbidity in a population of elderly men. J Hypertens 2004; 22: 1691-1697.

3 Rothwell PM. Limitations of the usual blood-pressure hypothesis and importance of variability, instability, and episodic hypertension. Lancet 2010; 375: 938-948.

4 Rothwell PM, Howard SC, Dolan E, O'Brien E, Dobson JE, Dahlöf B, Sever PS, Poulter NR. Prognostic significance of visit-to-visit variability, maximum systolic blood pressure, and episodic hypertension. Lancet 2010; 375: 895-905.

5 Pickering TG, James GD. Ambulatory blood pressure and prognosis. J Hypertens 1994; 12 (S8): S29-S33.

6 Eguchi K, Ishikawa J, Hoshide S, Pickering TG, Schwartz JE, Shimada K, Kario K. Night time blood pressure variability is a strong predictor for cardiovascular events in patients with type 2 diabetes. Am J Hypertens 2009; 22: 46-51.

7 Ozawa M, Tamura K, Okano Y, Matsushita Y, Yanagi M, Tsurumi-Ikeya Y, Oshikawa J, Hashimoto T, Masuda S, Wakui H, Shigenaga Al, Azuma K, Ishigami T, Toya Y, Ishikawa $\mathrm{T}$, Umenura S. Identification of an increased short-term blood pressure variability on ambulatory blood pressure monitoring as a coronary risk factor in diabetic hypertensives. Clin Exp Hypertens 2009; 31: 259-270.

8 Lucini D, Cusumano G, Bellia A, Kozakova M, Difede G, Lauro R, Pagani M. Is reduced baroreflex gain a component of the metabolic syndrome? Insights from the LINOSA study. J Hypertens 2006; 24: 361-370.

9 London GM, Schmieder R, Calvo C, Asmar R. Indapamide SR versus candesartan and amlodipine in hypertension: the X-CELLENT Study. Am J Hypertens 2006; 19: 113-119.

10 O'Brien E, Asmar R, Beilin L, Imai Y, Mallion JM, Mancia G, Mengden T, Myers M, Padfield P, Palatini P, Parati G, Pickering T, Redon J, Staessen J, Stergiou G, Verdecchia P. European Society of Hypertension recommendations for conventional, ambulatory and home blood pressure measurement. J Hypertens 2003; 21: 821-848.

11 Coats AJ, Conway J, Sleight P, Meyer TE, Somers VK, Floras JS, Vann Jones J. Interdependence of blood pressure and heart period regulation in mild hypertension. Am J Hypertens 1991; 4: 234-238.

12 Lucini D, Mela GS, Malliani A, Pagani M. Impairment in cardiac autonomic regulation preceding arterial hypertension in humans: insights from spectral analysis of beat-bybeat cardiovascular variability. Circulation 2002; 106: 2673-2679.

13 Lucini D, Zuccotti G, Malacarne M, Scaramuzza A, Riboni S, Palombo C, Pagani M. Early progression of the autonomic dysfunction observed in pediatric type 1 diabetes mellitus. Hypertension 2009; 54: 987-994.

14 Baldridge BR, Burgess DE, Zimmerman EE, Carroll JJ, Sprinkle AG, Speakman RO, Li SG, Brown DR, Taylor RF, Dworkin S, Randall DC. Heart rate-arterial blood pressure relationship in conscious rat before vs. after spinal cord transaction. Am J Physiol Regul Integr Comp Physiol 2002; 283: R748-R756.

15 Eguchi K, Schwartz JE, Pickering TG, Hoshide S, Ishikawa J, Shimada K, Kario K. Increased heart rate variability during sleep is a predictor for future cardiovascular events in patients with type 2 diabetes. Hypertens Res 2010; 33: 737-742.

16 Ichihara A, Kaneshiro Y, Takemitsu T, Sakoda M, Hayashi M. Ambulatory blood pressure variability and brachial-ankle pulse wave velocity in untreated hypertensive patients. J Hum Hypertens 2006; 20: 529-536.

17 Webb AJ, Fischer U, Mehta Z, Rothwell PM. Effects of antihypertensive-drug class on interindividual variation in blood pressure and risk of stroke: a systematic review and meta-analysis. Lancet 2010; 375: 906-915. 